\title{
Implementation of 6LoWPAN and Its Application in Smart Lighting
}

\author{
Zucheng Huang, Feng Yuan \\ Institute of Software Application Technology, Guangzhou \& Chinese Academy of Sciences (GZIS), Guangzhou, \\ China \\ Email: zucheng10@qq.com
}

Received January 2015

Copyright (C) 2015 by authors and Scientific Research Publishing Inc.

This work is licensed under the Creative Commons Attribution International License (CC BY).

http://creativecommons.org/licenses/by/4.0/

c) (i) Open Access

\begin{abstract}
Smart lighting system based on PLC (Power Line Communication) is lack of extensibility due to low data rate and non-standard communication protocol. 6LoWPAN is an IP-based communication standard for WSN (Wireless Sensor Network) proposed by IETF. We upgraded the SEMS smart lighting system from PLC to 6LoWPAN, PLC nodes were replaced by 6LoWPAN nodes and centralized controllers were replaced by border routers. 6LoWPAN system testing was carried out on the street after the implementation. The results show that smart lighting system based on 6LoWPAN is better than PLC in transmission rate, signal coverage range, compatibility and extensibility.
\end{abstract}

Keywords

6LoWPAN, Smart Lighting, PLC, WSN

\section{Introduction}

Smart lighting is a key area in the smart city construction nowadays. The Smart Energy Management System (SEMS) [1] built by Institute of Software Application Technology Guangzhou \& Chinese Academy of Sciences (ISCAS) uses PLC link to connect light controller nodes and the centralized controllers. Intellistreets developed a wireless street light control system with the functionality of remote lighting control, notification display and street surveillance. The Street Light Vision (SLV) created Central Management Software (CMS) which supports street light remote control via smart phones. All these systems have a common characteristic that they use a gateway to connect light controller nodes and the system server.

An increasing number of manufactures take part in the competition of the smart lighting industry. The consequence is that a huge amount of communication patterns is implemented in different brands of products, such as KNX [2], BACnet [3], DALI [4], ZigBee-ZHA/ZBA [5], PLC-Lonworks [6], etc. One critical problem comes 
up in all these products is that they cannot interoperate with each other (i.e., low compatibility and extensibility). So, this paper is focus on bring up a new solution 6LoWPAN [7] in smart lighting with the properties of high compatibility and extensibility.

The SEMS is a typical smart lighting system of the existing type. The fallowing sections will describe the architecture of SEMS which is based on PLC, and how it is upgraded to 6LoWPAN. Full performance test was carried out in the real street environment after the 6LoWPAN implementation. Based on the test results, a comparison between 6LoWPAN and PLC will be described in the later section to show advancement of 6LoWPAN.

\section{The SEMS Smart Lighting System}

The SEMS smart lighting system architecture contains three layers: sense layer, data process layer and application layer. Sense layer is made up of LED lights (the SEMS mainly uses LED for lighting), PLC light controllers and some sensors. LED is one of the energy saving and adjustable dimming street light. PLC light controllers work as terminal nodes in the system and provide data and/or events then report to the data server through a PLC link. Sensors are used for light sense and moving objects detection. Data process layer includes centralized controllers, data server and related transmission link. Centralized controllers are deployed in switch gears of each street block for data collection from all the terminal nodes within their communication range. Additionally, centralized controllers report all the lights' information to an associated data server through wireless network GPRS/3G or Internet depend on the network environment. Application layer is an end point user interface, it usually include PC(s), Pads and smart phones. The SEMS structure is shown in Figure 1.

From Figure 1 we can see that the SEMS smart lighting system consists of two types of networks, one is PLC network, and the other is Internet which is based on IP. These two types of networks are different in architecture, transmission media, link layer protocol and application protocol, so there must be a transformer between each network's borders. It is the centralized controller in this case. The centralized controllers forward PLC packets to the data server via Internet, they are crucial devices in the system.

This architecture has a drawback. If, for example, the functionality on the centralized controller had to be extended to support other applications like humidity and temperature collection, the centralized controller would have to support an additional software module. New software modules would then have to be added for each additional protocol that required support. Using this deployment model to address different application requirements presents fundamental challenges in terms of scalability and extensibility (e.g. field upgrade issues).

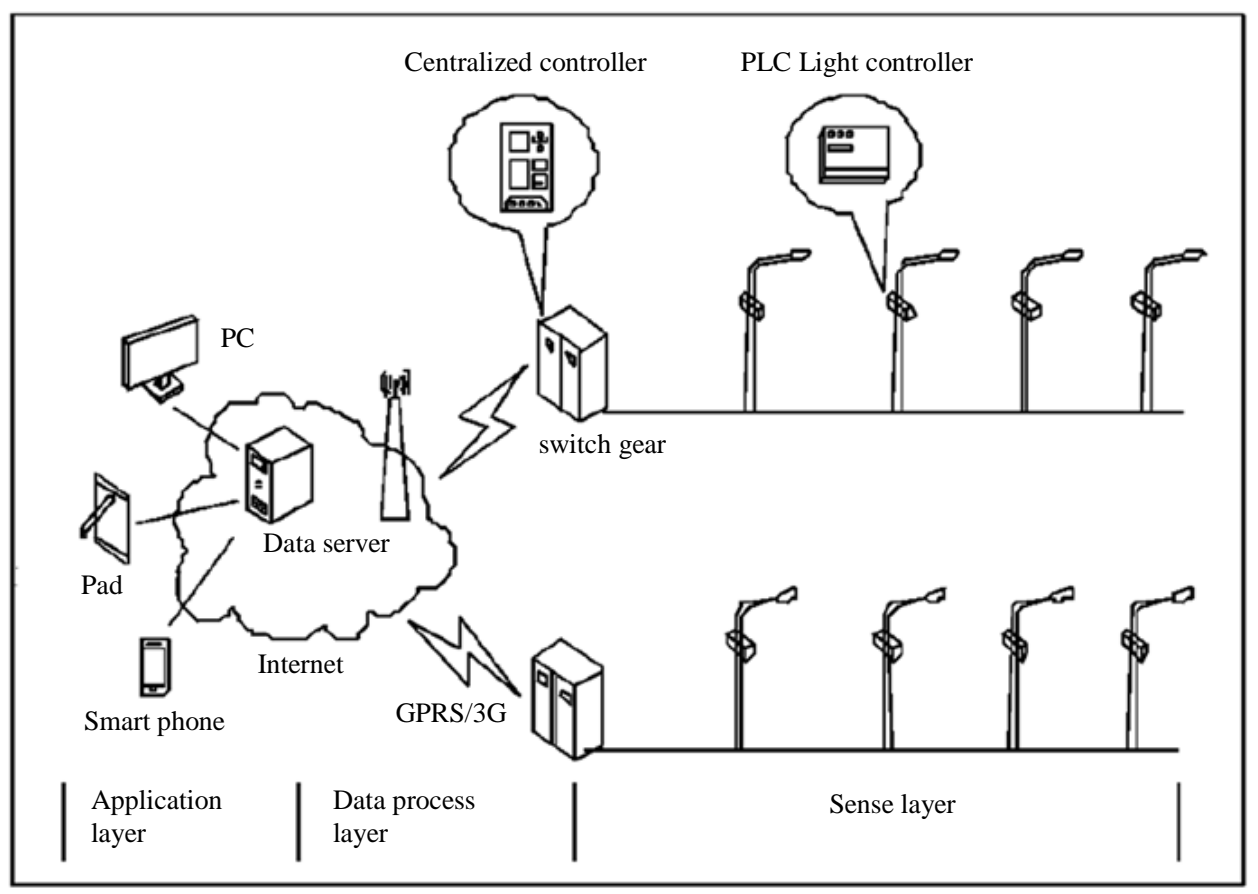

Figure 1. SEMS smart lighting system. 


\section{6LoWPAN Usage in Smart Lighting}

In the SEMS case, data process layer and application layer are both deployed in IP network. To make the system network architecture unified, the sense layer must support IP network. But IP protocol is not suitable for microcontroller and other low power wireless devices. Many standardization groups have made the effort in low power wireless network in the past (e.g., ZigBee, Z-Wave and Wireless HART), but their ad hoc solutions are not based on IP. In this case, different system cannot communicate with each other. In order to apply IP protocol in constrained wireless network, IETF published 6LoWPAN.

\subsection{LoWPAN Protocol Stack}

6LoWPAN protocol stack is similar to the TCP/IP protocol stack, the physical layer and MAC layer are subordinated to IEEE 802.15.4 standard, the adaption layer is named 6LoWPAN, network layer could be IPv6, ICMP or RPL (Routing Protocol for LLN), transmission layer runs UDP, and the application layer is CoAP (Constrained Application Protocol). The 6LoWPAN protocol stack is shown in Figure 2.

6LoWPAN usually runs a sub-network and communicate with other IP network through a border router. Border router uses IP/ICMP [8] or other IPv6 transmission mechanism to connect the 6LoWPAN and IPv4 [9] network as the 6LoWPAN only runs IPv6 based on IEEE 802.15.4. Border router does not need to maintain any application status or run any application protocol in the 6LoWPAN stack because it transfers packets in network layer.

\subsection{Smart Lighting with 6LoWPAN}

The Smart Lighting system with 6LoWPAN in this paper was upgraded from SEMS. PLC light controllers were replaced by 6LoWPAN light controllers and the centralized controllers were replaced by border routers. The system structure is shown in Figure 3.

In this implementation, CC2530 chips were used in light controllers. CC2530 is a SoC solution for low power wireless network based on IEEE 802.15.4, and it runs a light weight operating system Contiki, which developed by Adam Dunkels. Contiki integrate uIP protocol and 6LoWPAN stack, it provides BSD style Socket API, that makes it very easy to develop application based on IP communication. Border router contains an Atmel microprocessor AT91SAM9G45 which integrate with 10/100 Mb/s Ethernet interface and a GPRS model. The border router runs 6lbr routine, which forward 6LoWPAN packets to IPv6 or IPv4 network. By excluding centralized controllers in the smart lighting system, 6LoWPAN network reduces packets forwarding latency and increases throughput compare to the early PLC link.

\subsection{System Testing}

In order to simulate the 6LoWPAN smart lighting system, a testing model was built and the test was carried out in the real street environment. In this testing model, five 6LoWPAN nodes (C1 - C5) was installed on the street light poles, one border router was placed in one side of the street, the border router connected to a laptop computer via Ethernet link. The deployment of the testing system is shown in Figure 4.

\begin{tabular}{|c|c|c|c|}
\hline \multicolumn{3}{|c|}{ 6LoWPAN stack } & \\
\hline \multicolumn{3}{|c|}{ CoAP } & Application layer \\
\hline \multicolumn{3}{|c|}{ UDP } & Transmission layer \\
\hline Ipv6 & ICMP & RPL & Network layer \\
\hline \multicolumn{3}{|c|}{ 6LoWPAN } & Adaption layer \\
\hline \multicolumn{3}{|c|}{ IEEE 802.15.4 MAC } & MAC layer \\
\hline \multicolumn{3}{|c|}{ IEEE 802.15.4 PHY } & Physical layer \\
\hline
\end{tabular}

Figure 2. 6LoWPAN stack. 


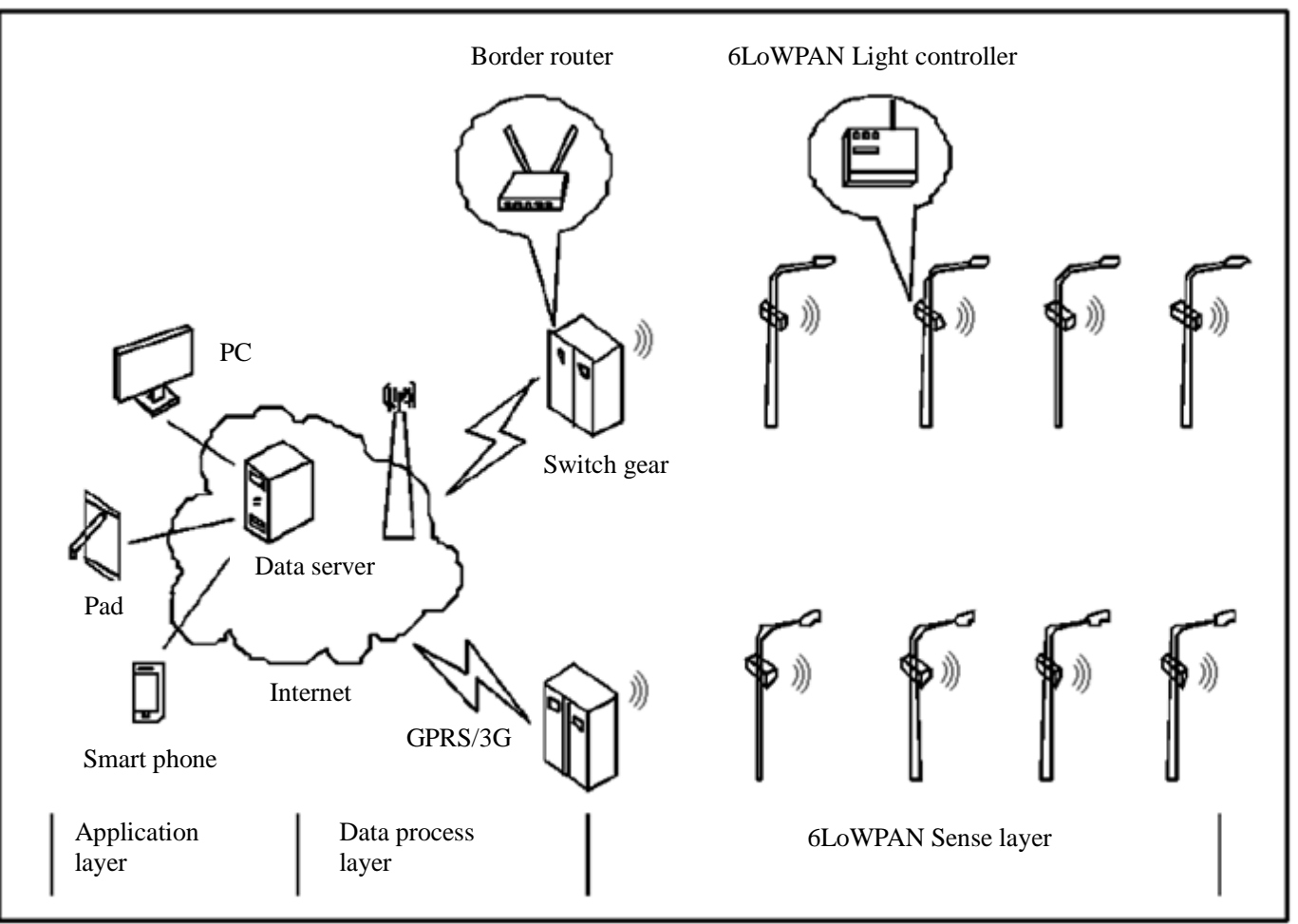

Figure 3. Smart lighting with 6LoWPAN.

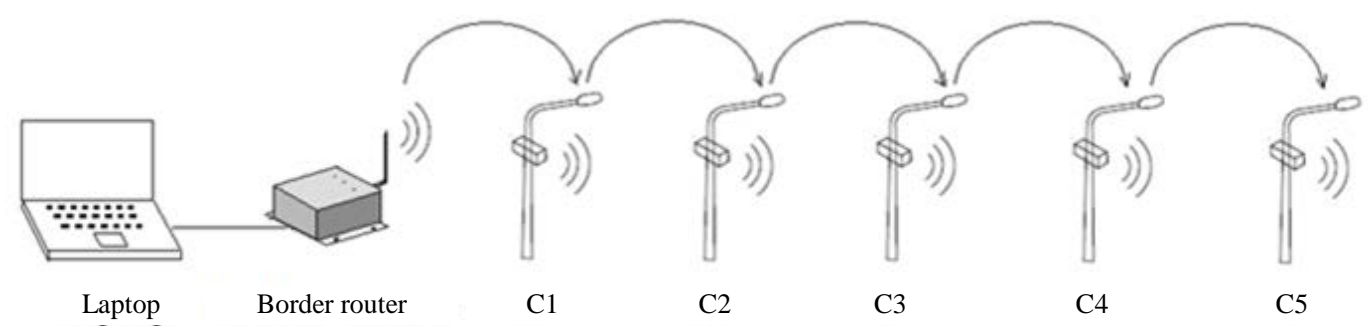

Figure 4. 6LoWPAN testing model.

The distance between each pole is about $30 \mathrm{~m}$. All these 6LoWPAN nodes were powered by lithium battery. Simulation software was ran on laptop computer and it sent constant packets to one node in a maximum rate to test the 6LoWPAN node's data receive capability. To test the node's reliability, the laptop sent packets to the nodes periodically, and nodes replied an ACK packet, the result is the proportion of sent and received packets. Each testing item was lasted for 10 minutes, and the reliability test for each node repeated three times. The test result is shown in Table $\mathbf{1 .}$

The test result from Table 1 shows that the average data rate is about 44 kbps and the reliability is $95 \%$ on average. The 6LoWPAN communication performance is good enough for the street light control and light status collection.

\section{Results and Discussion}

The SEMS solution is based on PLC chip form RISECOMM. A full test of the 6LoWPAN smart lighting system carried out after a complete review of the RISE3501E data manual [10]. The results show that 6LoWPAN is better than PLC in data rate, transmission range, reliability, anti-interference, etc. Dominant parameters of the PLC and 6LoWPAN in smart lighting system are listed in Table 2.

Besides the technical data, the preeminent advantage of deploying a 6LoWPAN-based smart lighting system is that one common application platform can be used for different application to gain access to the 6LoWPAN 
Table 1. Data rate and reliability of 6LoWPAN test.

\begin{tabular}{cccccc}
\hline Nodes & C1 & C2 & C3 & C4 & C5 \\
\hline Data rate (kbps) & 47.1 & 47.1 & 47.1 & 40.5 & 40.5 \\
& 100 & 100 & 97 & 100 & 84.6 \\
Reliability (\%) & 100 & 100 & 100 & 98.5 & 97 \\
& 98.5 & 100 & 100 & 84.6 & 98.5 \\
\hline
\end{tabular}

Table 2. Main Parameters of PLC and 6LoWPAN.

\begin{tabular}{ccc}
\hline Parameters & PLC & 6LoWPAN \\
\hline Data rate & $5 \mathrm{kbps}$ & $50 \mathrm{kbps}$ \\
Transmission range & $100 \mathrm{~m}$ & $300 \mathrm{~m}$ \\
Packets latency & $1-5 \mathrm{~s}$ & $<1 \mathrm{~s}$ \\
Interoperation & Centralized controller & Border router \\
Compatibility & Cannot integrate with different brands of node & All standard nodes are compatible \\
Security & None & AES-128 \\
Router protocol & No standard protocol & RPL \\
\hline
\end{tabular}

sensor data. This allows third-party developers leveraging back-end management systems to use standard 6LoWPAN interfaces to write productive new applications instead of having to learn a new application programming model each time a new protocol needs to be supported. This translates directly into ease-of-use, ease-of-deployment and accelerated time-to-market.

\section{Conclusions and Outlook}

From the above considerations we can see that implementing 6LoWPAN in smart lighting system gains the advantages in transmission rate, signal coverage range and compatibility compare to PLC. The smart lighting system based on 6LoWPAN with the standard IP-based interface will also make it much easier to integrate different types of sensors for environmental monitoring.

In this implementation, we only got five nodes in the testing model, and we are going to get more nodes for a large scale test in the next stage. Besides the lighting control nodes, we also want the system connecting to more sensors such as temperature sensors, humidity sensors, and air condition sensors to provide more environmental services.

\section{Acknowledgements}

This paper is supported by Lighting Control System Based on Cloud Computing Application Research on Key Techniques of Modular LED Street Lamp (No. 2013HK100062).

\section{References}

[1] Institute of Software Application Technology, Guangzhou \& Chinese Academy of Sciences (GZIS) (2014) Smart Energy Management System (SEMS). http://sems.gzis.ac.cn/

[2] Han, N., Wu, Y.-H. and Tang, Y. (2010) Research of KNX Device Node and Development Based on the Bus Interface Module. 29th Chinese Control Conference (CCC), 2010, 4346-4350.

[3] Park, T. and Hong, S.H. (2010) A New Proposal of Network Management System for BACnet and Its Reference Model. 8th IEEE International Conference on Industrial Informatics (INDIN), 2010, 28-33. http://dx.doi.org/10.1109/INDIN.2010.5549470

[4] Wohlers I., Andonov R. and Klau G.W. (2013) DALIX: Optimal DALI Protein Structure Alignment. IEEE/ACM 
Transactions on Computational Biology and Bioinformatics, 10, 26-36. http://dx.doi.org/10.1109/TCBB.2012.143

[5] Dominguez, F., Touhafi, A., Tiete, J. and Steenhaut, K. (2012) Coexistence with WiFi for a Home Automation ZigBee Product. IEEE 19th Symposium on Communications and Vehicular Technology in the Benelux (SCVT), 2012, 1-6. http://dx.doi.org/10.1109/SCVT.2012.6399392

[6] Lin, W.J., Wu, Q.X. and Huang, Y.W. (2009) Automatic Meter Reading System Based on Power Line Communication of LonWorks. International Conference on Technology and Innovation (ITIC 2009), 2009, 1-5. http://dx.doi.org/10.1049/cp.2009.1391

[7] Kushalnagar, N., Montenegro, G. and Schumacher, C. (2007) IPv6 over Low-Power Wireless Personal Area Networks (6LoWPANs): Overview, Assumptions, Problem Statement, and Goals. ITEF-RFC. http://www.rfc-editor.org/rfc/rfc4919.txt

[8] Nordmark, E. (2014) Stateless IP/ICMP Translation Algorithm (SIIT). http://www.rfc-editor.org/info/rfc2765

[9] Nordmark, E. and Gilligan, R. (2014) Basic Transition Mechanisms for IPv6 Hosts and Routers. http://www.rfc-editor.org/info/rfc4213

[10] (2014) RiseComm RISE3501E Data Manual. http://www.risecomm.com.cn/ 